\title{
A Markerless Method for Genome Engineering in Zymomonas mobilis ZM4
}

\author{
Piyush Behari Lal1,2, Fritz M. Wells ${ }^{1,2}$, Yucai Lyu ${ }^{1,3}$, Indro N. Ghosh ${ }^{1,4}$, Robert Landick ${ }^{1,4,5,6}$ \\ and Patricia J. Kiley ${ }^{1,2,5 *}$
}

\begin{abstract}
${ }^{1}$ DOE Great Lakes Bioenergy Research Center, University of Wisconsin-Madison, Madison, WI, United States, ${ }^{2}$ Department of Biomolecular Chemistry, University of Wisconsin-Madison, Madison, WI, United States, ${ }^{3}$ College of Biological and Pharmaceutical Sciences, China Three Gorges University, Yichang, China, ${ }^{4}$ Department of Biochemistry, University of Wisconsin-Madison, Madison, WI, United States, ${ }^{5}$ Cell and Molecular Biology Graduate Training Program, University of Wisconsin-Madison, Madison, WI, United States, ${ }^{6}$ Department of Bacteriology, University of Wisconsin-Madison, Madison, WI, United States
\end{abstract}

OPEN ACCESS

Edited by:

Mingxiong He,

Biogas Institute of the Ministry

of Agriculture (CAAS), China

Reviewed by:

Guodong Luan,

Qingdao Institute of Bioenergy and Bioprocess Technology (CAS),

China

Dennis Ken Bideshi,

California Baptist University, United States

*Correspondence: Patricia J. Kiley pjkiley@wisc.edu

Specialty section:

This article was submitted to Microbiotechnology, Ecotoxicology and Bioremediation,

a section of the journal

Frontiers in Microbiology

Received: 07 August 2019 Accepted: 10 September 2019

Published: 11 October 2019

Citation:

Lal PB, Wells FM, Lyu Y,

Ghosh IN, Landick R and Kiley PJ

(2019) A Markerless Method

for Genome Engineering

in Zymomonas mobilis ZM4.

Front. Microbiol. 10:2216.

doi: 10.3389/fmicb.2019.02216
Metabolic engineering of the biofuel-producing Zymomonas mobilis is necessary if we are to unlock the metabolic potential present in this non-model microbe. Manipulation of such organisms can be challenging because of the limited genetic tools for iterative genome modification. Here, we have developed an efficient method for generating markerless genomic deletions or additions in Z. mobilis. This is a two-step process that involves homologous recombination of an engineered suicide plasmid bearing Z. mobilis targeting sequences and a subsequent recombination event that leads to loss of the suicide plasmid and a genome modification. A key feature of this strategy is that GFP expressed from the suicide plasmid allows easy identification of cells that have lost the plasmid by using a fluorescence activated cell sorter. Using this method, we demonstrated deletion of the gene encoding lactate dehydrogenase $(I d h)$ and the operon for cellulose synthase (bcs $A B C$ ). In addition, by modifying the plasmid design, we demonstrated targeted insertion of the crt/BE operon encoding a neurosporene biosynthetic pathway into the $Z$. mobilis genome without addition of any antibiotic resistance genes. We propose this approach will provide an efficient and flexible platform for improved genetic engineering of $Z$. mobilis.

Keywords: genome engineering of a non-model bacterium, green fluorescent protein, fluorescence activated cell sorting, recombineering suicide plasmid, biofuels, Zymomonas mobilis

\section{INTRODUCTION}

While the pace of research into genetic tools for model organisms has accelerated over the last half century, the development of tools for non-model bacteria has lagged behind. Zymomonas mobilis ZM4, an obligatory fermentative alpha-proteobacterium, is one such organism that lacks convenient tools for genetic engineering. This organism has garnered significant interest as a platform for the biosynthesis of biofuels because of its native ability to efficiently metabolize glucose to ethanol rather than to biomass (He et al., 2014; Yang et al., 2016; Wang et al., 2018). To optimize Z. mobilis' metabolism and unlock its full potential for industrial fermentation of compounds of interest, methods for quickly and efficiently removing undesirable genes and pathways and adding novel genes and pathways are needed. 
Existing genetic tools for Z. mobilis are not optimal for creation of strains with multiple engineered loci. A common approach to insertionally inactivate genes relies on host homologous recombination of an antibiotic resistance gene flanked by $Z$. mobilis DNA contained on a suicide plasmid, which, once integrated into the genome, can be screened for events leading to loss of delivery plasmid and gene of interest (Senthilkumar et al., 2004; Strazdina et al., 2018). This approach was enhanced by use of lambda red recombinase to increase the frequency of recombination events (Senthilkumar et al., 2004; Bo et al., 2013). However, the inability to remove the antibiotic resistance cassette quickly limits the number of modifications that can be made to a particular strain especially given the natural resistance of $Z$. mobilis to several antibiotics (Bochner et al., 2010). To overcome this problem, FLP recombinase was used to remove the antibiotic resistance gene to create a markerless gene deletion (Zou et al., 2012). However, introduction and removal of the stable plasmid bearing the FLP recombinase is a timeconsuming process. To avoid the problems associated with using antibiotic resistance genes, Xia et al. (2018) made use of a sucrose counter-selection $(s a c B)$ to accelerate the process of integrated plasmid loss (Xia et al., 2018), but Z. mobilis ZM4 is annotated to also have $s a c B$, questioning the general utility of this approach. Lastly, a CRISPR/Cas9 system was deployed to remove a native plasmid from Z. mobilis (Cao et al., 2017). This technique has not yet been extended to genome modification. Therefore, we sought to develop a flexible and an efficient method for genome engineering that would overcome some of the limitations of existing methods.

In this paper, we describe the creation of an efficient, reproducible, and flexible method for making markerless mutations in $Z$. mobilis by homologous recombination. We demonstrate its primary utility by making knockouts of the $l d h$ gene (ZMO0256: lactate dehydrogenase) and the $b c s A B C$ genes (ZMO1083-85; cellulose synthase). We also describe how this technique could be extended to make markerless knock-ins at a desired locus by introducing carotenoid biosynthetic genes, crtIBE, from Rhodobacter sphaeroides (Takaichi and Maoka, 2015; $\mathrm{Su}$ et al., 2018). Herein, we also describe the suicide vector, which forms the basis for this technique. We also describe our unique choice to include the gene encoding GFP in our vector, which, through the use of a transilluminator or fluorescence activated cell sorting (FACS), allows us to track and screen for the presence or absence of the plasmid during intermediate steps in the process. This system should allow the facile and targeted addition or deletion of genes of interest in an iterative manner and thus facilitate the engineering of $Z$. mobilis for desirable traits.

\section{MATERIALS AND METHODS}

Primers, bacterial strains, and plasmids used in this study are listed in Tables 1-3.

\section{Growth of Bacterial Strains}

Zymomonas mobilis strains were cultured in rich medium ZRMG ( $1 \%$ yeast extract, $0.2 \% \mathrm{KH}_{2} \mathrm{PO}_{4}, 2 \%$ glucose) or glucose-minimal
TABLE 1 | Primer list.

\begin{tabular}{|c|c|}
\hline $\begin{array}{l}\text { Primer } \\
\text { number }\end{array}$ & Primer sequence $\left(5^{\prime}-3^{\prime}\right)$ \\
\hline P1 & GTGCCGAGGATGACGATG \\
\hline P2 & CGTCATCCTCGGCACGATGACGATTGTGCCTITG \\
\hline P3 & CAGAAAGCCTAATAACCCATAGCCTTACG \\
\hline P4 & CGTAAGGCTATGGGTTATTAGGCTITCTG \\
\hline P5 & GCTACGCCTGAATAAGTGACCAGCAACTITATCAGAC \\
\hline P6 & ACTTATTCAGGCGTAGCAC \\
\hline P7 & GACCAGCAACTITATCAGAC \\
\hline P8 & GTCTGATAAAGTTGCTGGTCAGCGCTCGGTCTTGCCTT \\
\hline P9 & GGTGCTACGCCTGAATAAGTGCGCTITCCGCTGCATAAC \\
\hline P10 & GCGCTITCCGCTGCATA \\
\hline P11 & GTTATGCAGCGGAAAAGCGCATAGGCGTATCACGAGGC \\
\hline P12 & GGTGCTACGCCTGAATAAGTGTGCTTAAAACGCAAAAAGC \\
\hline P15 & ACCGAGCGCTACTAGTGATAAGCTGTCAAACATG \\
\hline P16 & TATCACTAGTAGCGCTCGGTCTTGCCTT \\
\hline P17 & AGCTTAACTAACTAGTTATATCGACC ПITATITCAAAAATTAATC \\
\hline P18 & GGTCGATATAACTAGTTAGTTAAGCTGCTATAAATATTATATIITAC \\
\hline P19 & GCAAGGCAAGACCGAGCGCTTCAAGACCAGCAACTTATC \\
\hline P20 & AGCGCTCGGTCTTGCCTT \\
\hline P21 & CGTTCGGCTGTTCTGGGTG \\
\hline P22 & AAACCCTGTAAGCCGGGAAGC \\
\hline P23 & TGGGCGTTTCCACTGTTGCG \\
\hline P24 & ATGTATATCTCCTTCTTAAAGTTAAAC \\
\hline P25 & TITAAGAAGGAGATATACATATGCCCTCGATCTCGCCC \\
\hline P26 & TATTATAATAGAAAGTAAAGACTAGATCGGGTTGGCCCG \\
\hline P27 & $\begin{array}{l}\text { CCCGATCTAGTCTIACПTCTATTATAAATAAAGGAGACC } \\
\text { TICATGAGGCACAAGATGGCGTITGACAGC }\end{array}$ \\
\hline P28 & GCAGGAATTCGATATCAAGCTCAGACGCGGGCCGCGAC \\
\hline P29 & GCTTGATATCGAATTCCTG \\
\hline P30 & ATACGCCTATTATATCGACCTITAПITCAAAAATTAATC \\
\hline P31 & GGTCGATATAATAGGCGTATCACGAGGCCCTाTCG \\
\hline P32 & TATAGCAGCTTAATCAGACGCGGGCCGCGAC \\
\hline P33 & CCGCGTCTGATTAAGCTGCTATAAATATTATATITACAAAATAATGC \\
\hline P34 & CGCTTGACCTITACTTCGCC \\
\hline P35 & CAGGCTCACCCGCTAAAGTC \\
\hline P36 & CGAAATACCAGACGAACAGCCC \\
\hline P37 & ATCTATGCGGGTATCGGCGAC \\
\hline P38 & TCAACACAGGCTTCAACGAC \\
\hline
\end{tabular}

medium (Martien et al., 2019) at $30^{\circ} \mathrm{C}$. Escherichia coli strains were grown in Luria-Bertani (LB) medium. Chloramphenicol was used at a final concentration of $120 \mu \mathrm{g} \mathrm{ml}^{-1}$ for $Z$. mobilis and $20 \mu \mathrm{g} \mathrm{ml}^{-1}$ for E. coli strains. Spectinomycin was used at a final concentration of $100 \mu \mathrm{g} \mathrm{ml}^{-1}$ for $Z$. mobilis and $50 \mu \mathrm{g} \mathrm{ml}^{-1}$ for E. coli strains.

\section{Conjugation}

Escherichia coli donor strain WM6026 (Blodgett et al., 2007) was used to conjugate plasmids into the recipient strain $Z$. mobilis ZM4. WM6026, a $m$-diaminopimelate (DAP) auxotrophic strain, was grown at $30^{\circ} \mathrm{C}$ aerobically in LB containing DAP $(0.1 \mathrm{mM})$ with appropriate antibiotic. Overnight cultures were then subcultured into the same medium but lacking antibiotic to an $\mathrm{OD}_{600}$ of 0.15 and were grown to an $\mathrm{OD}_{600}$ of 0.5 . Z. mobilis 
TABLE 2 | Strain list.

\begin{tabular}{|c|c|c|}
\hline Strain & Description & Source or references \\
\hline WM6026 & 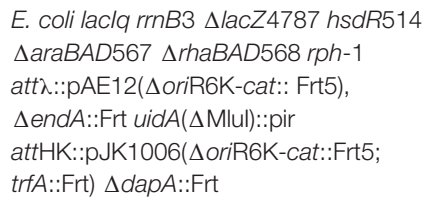 & Blodgett et al., 2007 \\
\hline $\mathrm{DH} 5 \alpha$ & $\begin{array}{l}\text { E. coli } \mathrm{F}^{-} \text {endA1 glnV44 thi-1 recA1 } \\
\text { relA1 gyrA96 deoR nupG purB20 } \\
\varphi 80 \mathrm{~d} / \mathrm{ac} Z \Delta \mathrm{M} 15 \Delta(\mathrm{lac} Z Y A \text {-argF }) \cup 169 \\
\left.\text { hsdR17( } \mathrm{r}_{\mathrm{K}}^{-} \mathrm{mK}_{\mathrm{K}}^{+}\right), \lambda^{-}\end{array}$ & Lab collection \\
\hline Z. mobilis & Zymomonas mobilis ZM4 ATCC 31281 & GLBRC \\
\hline PK15556 & Z. mobilis IdhuP::pPK15552 & This work \\
\hline PK15557 & Z. mobilis IdhuP::pPK15535 & This work \\
\hline PK15569 & Z. mobilis IdhuP::pPK15564 & This work \\
\hline PK15575 & Z. mobilis $\Delta / d h:: c r t I E B$ & This work \\
\hline PK15589 & Z. mobilis bcsABC $\mathrm{UP}:: \mathrm{pPK} 15538$ & This work \\
\hline PK15597 & Z. mobilis $\triangle b c s A B C$ & This work \\
\hline PK15598 & Z. mobilis $\Delta / d h$ & This work \\
\hline
\end{tabular}

TABLE 3 | Plasmid list.

\begin{tabular}{|c|c|c|}
\hline Plasmid & Description & $\begin{array}{l}\text { References or } \\
\text { source }\end{array}$ \\
\hline pRL814 & Broad host range plasmid & Ghosh et al., 2019 \\
\hline pACYC184 & cat $\mathrm{p} 15 \mathrm{~A}$ ori tet & Lab collection \\
\hline pPK15252 & $\begin{array}{l}\text { Gibson assembly of } \mathrm{p} 15 \mathrm{~A} \text { ori and cat } \\
\text { ( } 2115 \text { bp of pACYC184 amplified by } \\
\text { primers } \mathrm{P}_{1} \text { and } \mathrm{P}_{6} \text { ), } 500 \text { bp flanking } \\
\text { regions of } Z \text {. mobilis Idh (Idh } h_{\mathrm{UP}} \text { and } \\
\text { Idh }_{\mathrm{DN}} \text { fragments) }\end{array}$ & This work \\
\hline pPK15296 & $\begin{array}{l}\text { pPK15252 with mob from pSUP202 } \\
\text { inserted between cat and } / d h_{\mathrm{DN}}\end{array}$ & This work \\
\hline pPK15303 & $\begin{array}{l}\text { pPK15296 with RBS element and gfp } \\
\text { gene from pRL814 inserted between } \\
\text { cat and mob }\end{array}$ & This work \\
\hline pPK15445 & pPK15303 with optimized RBS for gfp & This work \\
\hline pPK15534 & $\begin{array}{l}\text { pPK15445 with deletion of terminal } \\
100 \text { bp of p15A fragment to remove tet } \\
\text { promoter and adjacent Id } h_{\mathrm{UP}} \text { and } / d h_{\mathrm{DN}} \\
\text { replaced by a Spel site }\end{array}$ & This work \\
\hline pPK15535 & $\begin{array}{l}\text { pPK15445 with deletion of terminal } \\
100 \text { bp of p15A fragment to remove tet } \\
\text { promoter and insertion of Spel site } \\
\text { between } / d h_{\mathrm{UP}} \text { and } / d h_{\mathrm{DN}}\end{array}$ & This work \\
\hline pPK15538 & $\begin{array}{l}\text { pPK15534 with } 500 \text { bp regions } \\
\text { upstream and downstream of the } \\
\text { bcsABC operon inserted at Spel site }\end{array}$ & This work \\
\hline pPK15564 & $\begin{array}{l}\text { pPK15535 with crtIEB cassette inserted } \\
\text { at Spel site between } / d h_{\mathrm{UP}} \text { and } / d h_{\mathrm{DN}}\end{array}$ & This work \\
\hline pPK15552 & $\begin{array}{l}\text { pPK15303 with deletion of terminal } \\
100 \text { bp of p15A fragment to remove tet } \\
\text { promoter }\end{array}$ & This work \\
\hline pSUP202 & pMB1 replicon, $\mathrm{Ap}^{\mathrm{R}}, \mathrm{Tc}^{\mathrm{R}}, \mathrm{Cm}^{\mathrm{R}}$, mob & Simon et al., 1983 \\
\hline
\end{tabular}

was incubated in $5.0 \mathrm{ml}$ of ZRMG without shaking at $30^{\circ} \mathrm{C}$ until late exponential phase was reached. For conjugation, $1.0 \mathrm{ml}$ of cells, adjusted to $\mathrm{OD}_{600}$ of 0.5 , of both recipient and donor was mixed in a $2.0 \mathrm{ml}$ microfuge tube. The cell suspension was briefly centrifuged at $17,300 \times g$ for $30 \mathrm{~s}$, and the supernatant was decanted. The pellet was resuspended in the small amount of remaining medium and placed as a drop on a prewarmed (30$37^{\circ} \mathrm{C}$ ) ZRMG containing $1 \%$ tryptone, $0.15 \mathrm{mM}$ DAP agar plate. Plates were incubated overnight at $30^{\circ} \mathrm{C}(12-15 \mathrm{~h})$. Cells were collected from plates by resuspending into $1.0 \mathrm{ml}$ ZRMG liquid media. The conjugation mixture was then vortexed for $10 \mathrm{~s}$, spun at $17,300 \times g$, and the pellet was resuspended in $1.0 \mathrm{ml}$ ZRMG liquid medium. The suspensions were incubated without shaking at $30^{\circ} \mathrm{C}$ for $2 \mathrm{~h}$, and $100 \mu \mathrm{l}$ of the undiluted, 10 -fold, and 100 fold diluted cell suspension was plated on antibiotic-containing ZRMG agar plates. Plates were incubated at $30^{\circ} \mathrm{C}$.

\section{Enrichment of Non-fluorescent Cells Using FACS}

A single fluorescent exconjugant colony was grown in liquid ZRMG medium at $30^{\circ} \mathrm{C}$ without shaking. Twenty microliters of cell suspension from early log phase was diluted in $1 \mathrm{ml}$ sterile $137 \mathrm{mM} \mathrm{NaCl}, 2.7 \mathrm{mM} \mathrm{KCl}, 10 \mathrm{mM} \mathrm{Na}_{2} \mathrm{HPO}_{4}, 1.8 \mathrm{mM}$ $\mathrm{KH}_{2} \mathrm{PO}_{4}$, pH 7.4, and loaded into a Sony MA900 or SH800 FACS equipped with a $70 \mu \mathrm{m}$ sorting chip according to the manufacturer's recommendations. Sorting of non-fluorescent events away from the major population of fluorescent cells was done using a $488 \mathrm{~nm}$ laser for excitation and 525/50 $\mathrm{nm}$ detector to measure emission. Sorted events were collected in $1 \mathrm{ml} \mathrm{ZRMG}$ liquid medium, a fraction of which was plated on ZRMG agar plates. Alternatively, one can screen for $g f p^{-}$cells by plating the recombinant cell suspension, grown without selection, on antibiotic-free rich medium plates to obtain $\sim 10,000$ colonies and visually screening colonies that have lost fluorescence with a blue light transilluminator.

\section{PCR Amplification of DNA}

For PCR amplification of DNA fragments to be used for cloning or sequence verification, the high fidelity Q5 polymerase from New England Biolabs was used. For screening or confirming genome modifications, GOTaq ${ }^{R}$ Flexi DNA polymerase (Promega) was used. For colony PCR, a single colony was picked and resuspended in $50.0 \mu \mathrm{l}$ of distilled water. $2.0 \mu \mathrm{l}$ of the suspension was used as a template for PCR amplification. Cell lysis was achieved by heating the PCR reaction mixture at $98^{\circ} \mathrm{C}$ for $30 \mathrm{~s}$ in a thermocycler before amplification steps. Annealing temperature and elongation time were followed as directed by the manufacturer's specifications. Standard techniques were used for analysis or purification of DNA fragments or other molecular biology methods.

\section{Construction of Suicide Plasmid (pPK15534)}

A conjugatable suicide plasmid (pPK15534) was constructed in several steps by PCR amplification of indicated DNA fragments followed by Gibson assembly using NEBuilder ${ }^{\circledR}$ HiFi DNA Assembly Cloning Kit. DNA fragments containing the p15A origin of replication (ori) and a chloramphenicol resistance gene (cat) were amplified from pACYC184 (Chang and Cohen, 1978), and 500 bp regions upstream (ldh $\left.h_{\mathrm{UP}}\right)$ and downstream $\left(l d h_{\mathrm{DN}}\right)$ of the $l d h$ coding region were amplified from the 
Z. mobilis genome, using primer pairs P1-P6, P2-P3, and P4$\mathrm{P} 5$, respectively, to generate $\mathrm{PPK} 15252$. A plasmid mobilization element, mob, was amplified from pSUP202 (Simon et al., 1983) using primers P8-P9 and cloned at the junction of $l d h_{\mathrm{DN}}$ and cat in pPK15252, amplified by primers P7-P6 to generate $\mathrm{pPK}$ 15296. A DNA fragment encoding the superfolder fluorescent protein, GFP, driven by the T7A1 promoter and a ribosome binding site was amplified from pRL814 (Ghosh et al., 2019) using primers P11-P12 and added to pPK15296, amplified by primers P6-P10 to generate pPK15303. The ribosome binding site was subsequently optimized using Salis' algorithm (Salis et al., 2009; Borujeni et al., 2014) to improve GFP fluorescence in Z. mobilis by PCR amplification of PPK15303 using primers P13 and P14, which contain optimized RBS sequence in overhang regions to generate pPK15445. An orphan tet promoter was also removed and SpeI cloning site was introduced by Gibson assembly of two fragments of pPK15445 generated by a pair of primers P19-P18 and P17-P20, to generate pPK15535. Finally, to create a vector for cloning any target DNA (pPK15534), the orphan tet promoter, $l d h_{\mathrm{UP}}$ and $l d h_{\mathrm{DN}}$, were removed and replaced by a SpeI cloning site by Gibson assembly of a fragment of pPK15445 generated by a pair of primers P15-P16.

\section{Measurement of the Carotenoid Neurosporene}

To measure the production of neurosporene, we followed an established protocol (Wraight et al., 1978). Briefly, cells were grown in ZRMG media to an $\mathrm{OD}_{600}$ of 0.5 , the cell pellet from $2 \mathrm{ml}$ culture was resuspended in $100 \mu \mathrm{l}$ water, mixed with $1 \mathrm{ml}$ of 7:2 mixture of acetone:methanol solvent, and spun for $3 \mathrm{~min}$ at $21,000 \times g$. The absorbance between 300 and $550 \mathrm{~nm}$ of the resulting supernatant was recorded on a Perkin Elmer Lambda 25 spectrophotometer.

\section{RESULTS}

\section{Overview}

To create a more flexible system for deleting or adding genes iteratively to $Z$. mobilis, we rationalized that a recombinationbased method was needed that did not require permanent introduction of antibiotic resistance genes into the genome (i.e., markerless modifications) but still could be rapidly screened for desired events. Creation of a conjugatable suicide vector containing GFP (pPK15534) allowed a more convenient method for tracking the loss of the recombineering suicide plasmid from strains, resulting in markerless gene deletions or insertions. Here, we describe the development and function of this system with three examples.

\section{Demonstration of an Efficient Conjugation System for Introducing DNA Into Z. mobilis}

To implement a gene-modification system our first step was to establish a reproducible and efficient conjugation method for introducing plasmids into Z. mobilis from E. coli. We took advantage of an existing $E$. coli donor strain that possesses a chromosomally encoded copy of the RP4 conjugation machinery able to transfer plasmids containing a mobilization element (mob) and is auxotrophic for DAP (Blodgett et al., 2007). When this E. coli donor strain containing the broad host range plasmid pRL814 was mated with $Z$. mobilis for an optimum period of $12-14 \mathrm{~h}$ at $30^{\circ} \mathrm{C}$ on a solid medium (Figure 1), we found a reproducible transfer frequency of $0.1 \%$ per input recipient cells; conjugations performed in liquid medium were generally unsuccessful, which was expected since conjugation by RP4 (IncP-type) machinery is enhanced on solid medium (Bradley et al., 1980). Importantly, this conjugation efficiency was robust enough to support the use of a suicideplasmid homologous-recombination-based integration method; exconjugants should appear at a 100-1000-fold less frequency than that obtained with a stable plasmid to account for the additional recombination event.

\section{Designing a Mobilizable Suicide Plasmid pPK15534 for Z. mobilis}

We designed the suicide plasmid to easily track genomic recombination events (Figure 2). The origin of replication, oriC, was selected from pACYC184, which was known not to replicate in $Z$. mobilis. The RP4 mob element from pSUP202 was chosen to enable conjugation from an $E$. coli donor strain (Simon et al., 1983). A single SpeI restriction site was introduced to facilitate cloning of target DNA to direct recombination of the plasmid into the Z. mobilis genome. Inclusion of cat on the plasmid backbone allowed selection of chloramphenicol resistance to identify single crossover recombination events in $Z$. mobilis. The superfolder $g f p$ (Pedelacq et al., 2006), also on the plasmid
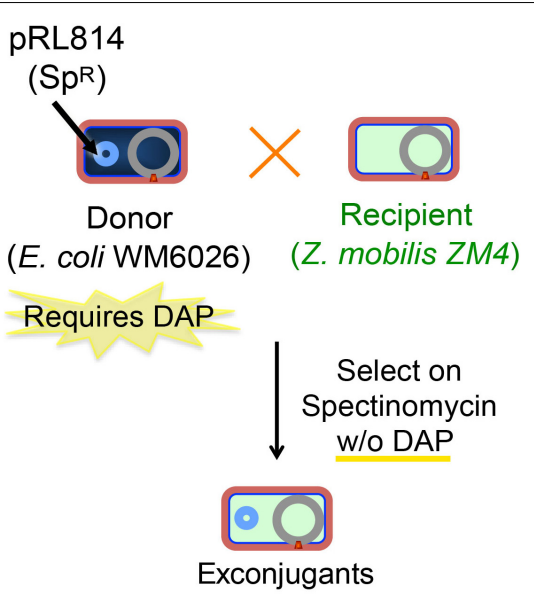

FIGURE 1 | Scheme for conjugation of plasmids from E. coli to Z. mobilis. Z. mobilis (light blue) was conjugated using the DAP auxotrophic E. coli strain WM6026 (dark blue) as described in the section "Materials and Methods." Briefly, donor and recipient strains were mixed and incubated in ZRMG containing tryptone and DAP overnight at $30^{\circ} \mathrm{C}$. The conjugation mixture was recovered in liquid ZRMG medium at $30^{\circ} \mathrm{C}$ for $2 \mathrm{~h}$, and plated in ZRMG with appropriate antibiotic. 

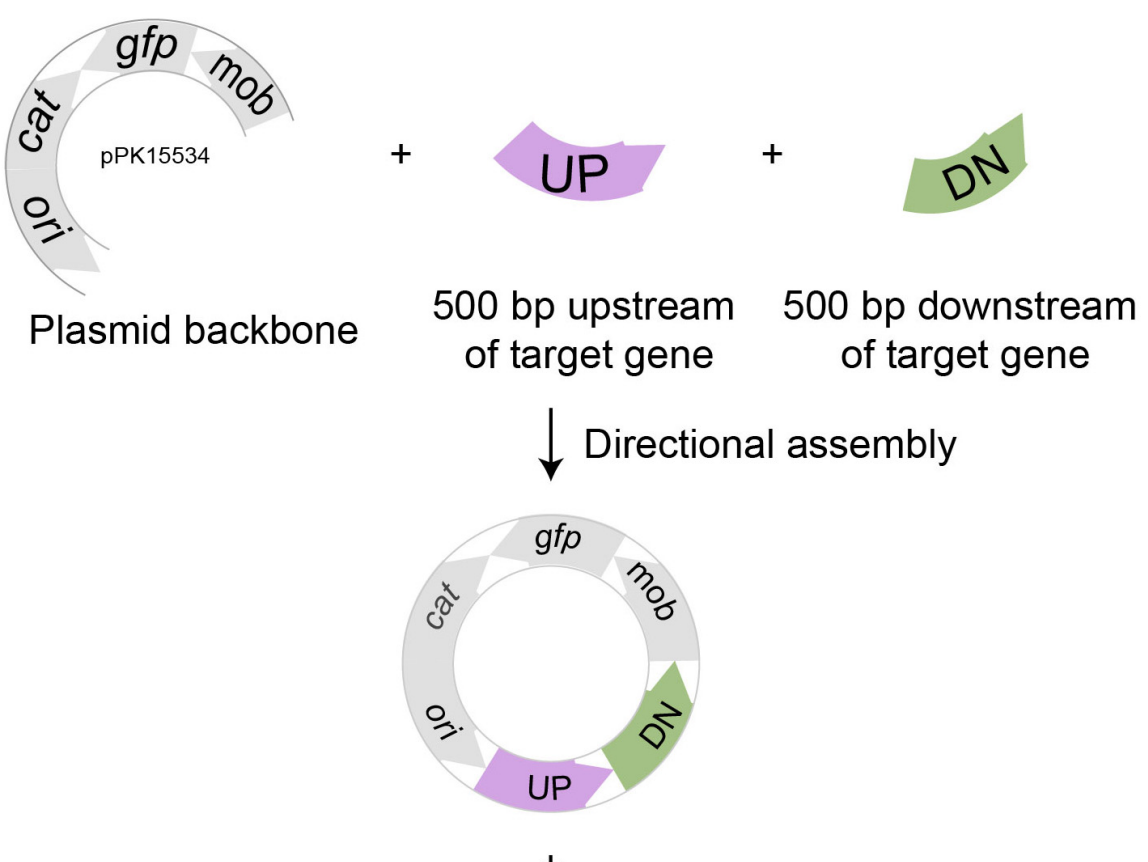

\section{Z. mobilis genome}

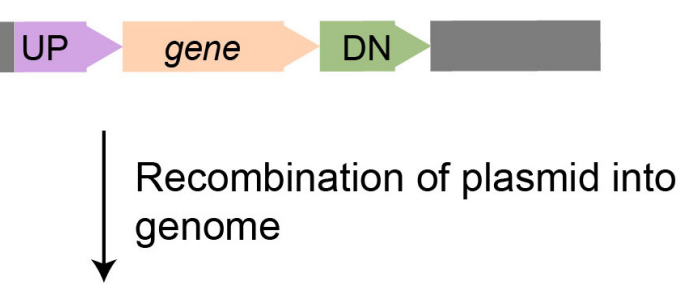

Recombination at UP region

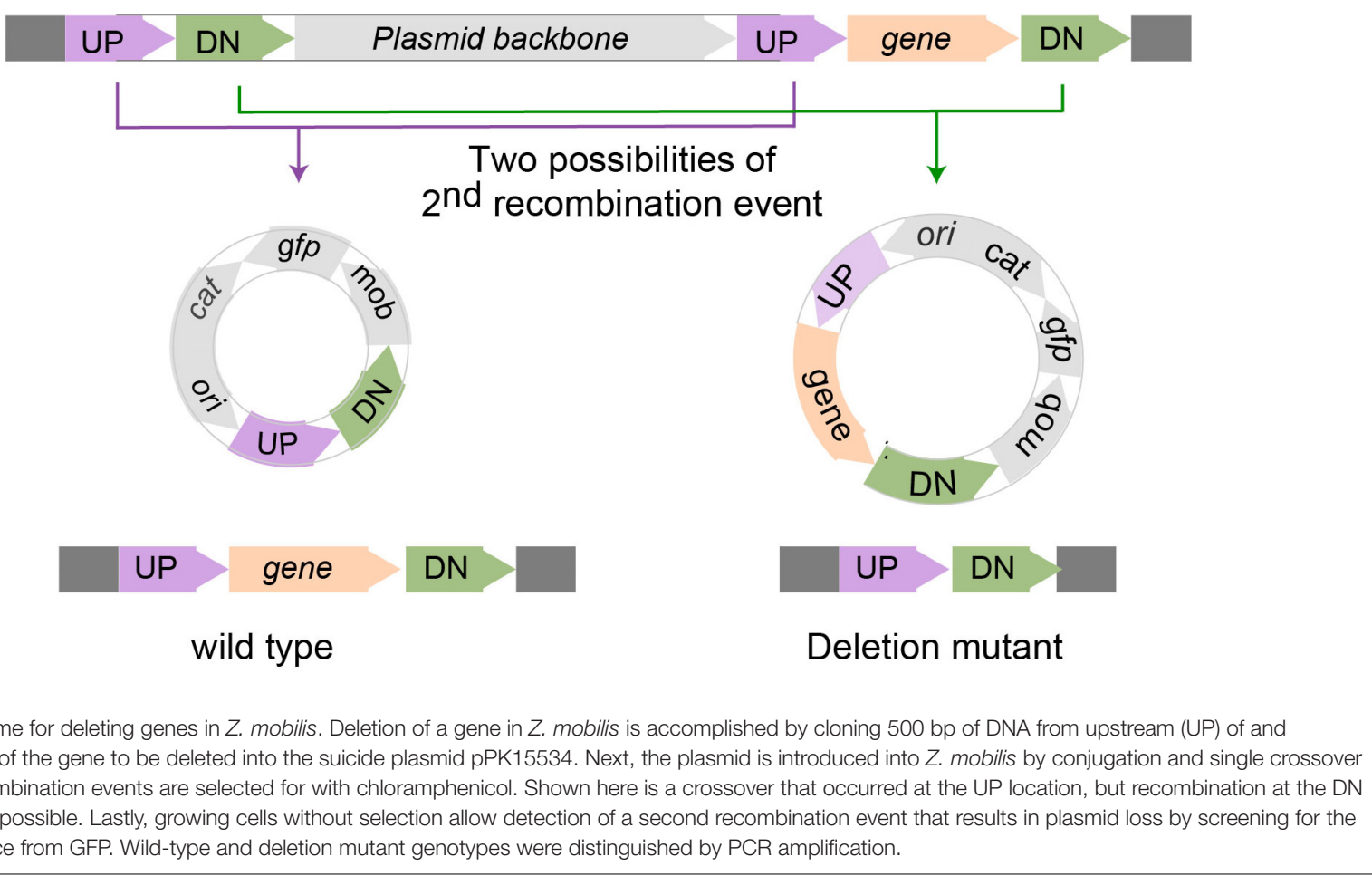


backbone, allowed direct tracking of the plasmid and sorting by FACS. The latter enabled rapid enrichment of non-fluorescent cells that had undergone additional recombination events and lost the plasmid.

\section{pPK15534 Can Be Used as a Vehicle to Efficiently Delete Genes in Z. mobilis}

As a proof of principle, we tested whether we could efficiently delete an average size gene, choosing the $1.7 \mathrm{~kb}$ ldh gene encoding lactate dehydrogenase (ldh, ZMO0256), since its function should be dispensable in Z. mobilis (Zou et al., 2012; Strazdina et al., 2018; Jones-Burrage et al., 2019). Our suicide plasmid (pPK15534) was engineered to contain two 500 bp sequences, immediately flanking but not including the target gene (pPK15535) (Figure 2). These sequences should direct homologous recombination of the plasmid into the genome, crossing over either upstream or downstream of the target gene within the genome. Following conjugation of the plasmid into Z. mobilis, we isolated $\mathrm{Cm}^{R}$ exconjugants at a frequency of $10^{-5}$, a reasonable frequency for a single recombination event following conjugation. We confirmed the integration location of
A

Grow recombinants

for several generations

without selection

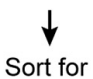

non-fluorescent cells (B) and plate on non-selective medium

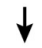

Verify colonies for the loss of fluorescence (C) $\downarrow$ PCR to identify the deletion strain (D-E)

D

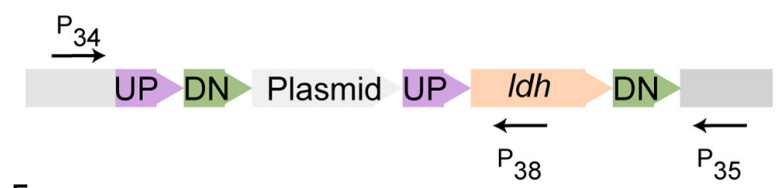

E

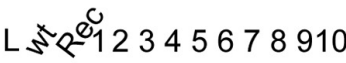

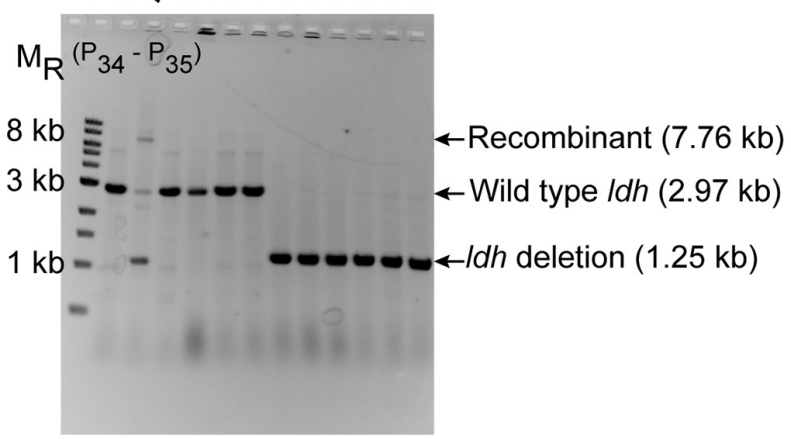

B

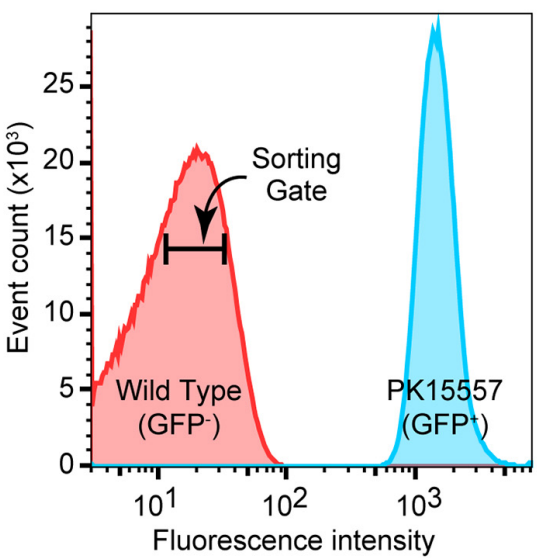

C

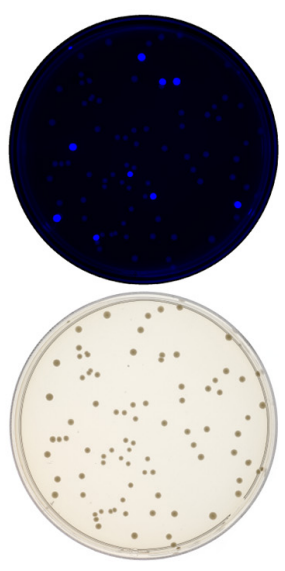

\section{$\mathbf{F}$}

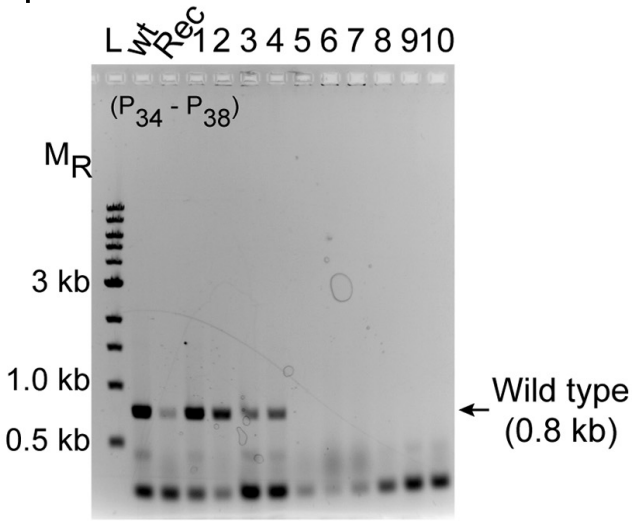

FIGURE 3 | Deletion of Idh gene. (A) Workflow for enrichment of non-fluorescent cells and screening for the modified genome. (B) Plot from FACS of Z. mobilis strain PK15557 that has suicide plasmid (pPK15535) recombined into the genome. The $X$-axis represents fluorescence intensity and $Y$-axis represents the total number of sorted events. The sorting gate for collecting the non-fluorescent cells (red peak) is indicated by the bar. (C) Top plate shows the fluorescence image of colonies from FACS sorted non-fluorescent cells plated on non-selective media. Bottom plate shows the same colonies viewed with visible light. (D) The location of primers used to screen non-fluorescent candidates for wild-type (wt) and deletion genotypes by PCR is indicated by arrows. (E,F) Agarose gel electrophoresis of products from PCR amplification of 10 non-fluorescent-candidate colonies. Selected size markers are indicated on the left ( $M_{R}$ ). (E) Amplification using primers P34 and P35 yielded a 1.25-kb DNA fragment for the Idh deletion whereas the wt Idh DNA fragment was $2.97 \mathrm{~kb}$. Strains that still possess the plasmid integrated into the genome (Rec) are expected to have a band of $7.8 \mathrm{~kb}$. (F) Amplification using P34 and P38 yielded a DNA fragment of $0.8 \mathrm{~kb}$, confirming those strains that still possess the Idh gene, while strains in which Idh has been deleted (six total) lacked the equivalent amplified fragment. Labels are the same as in panel E. Similar results were obtained when this experiment was biologically replicated three times. 


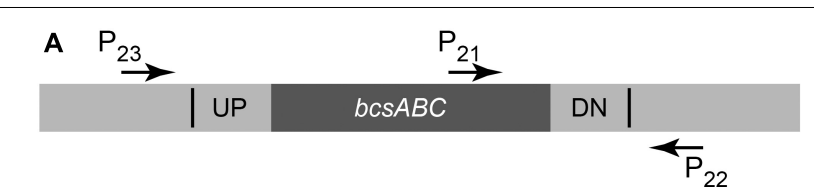

B

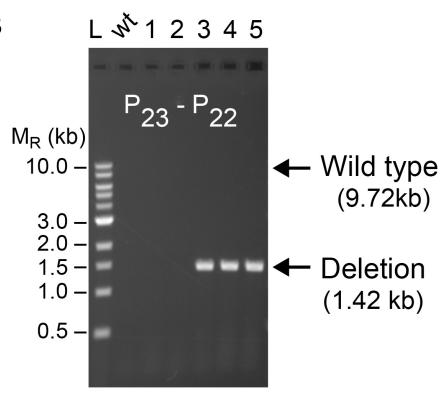

C

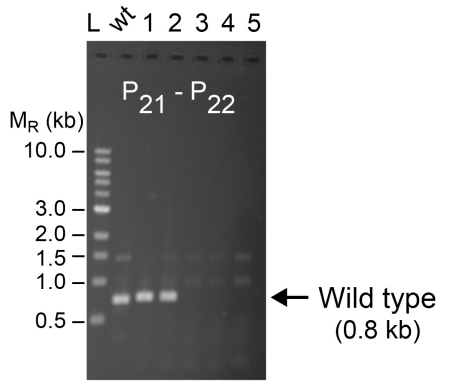

D

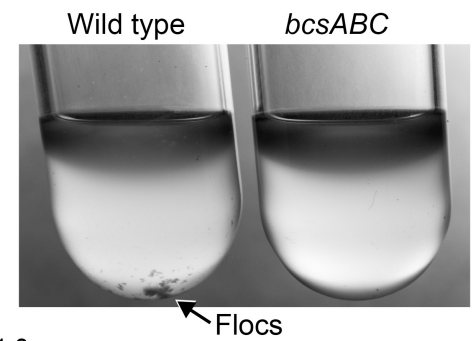

E

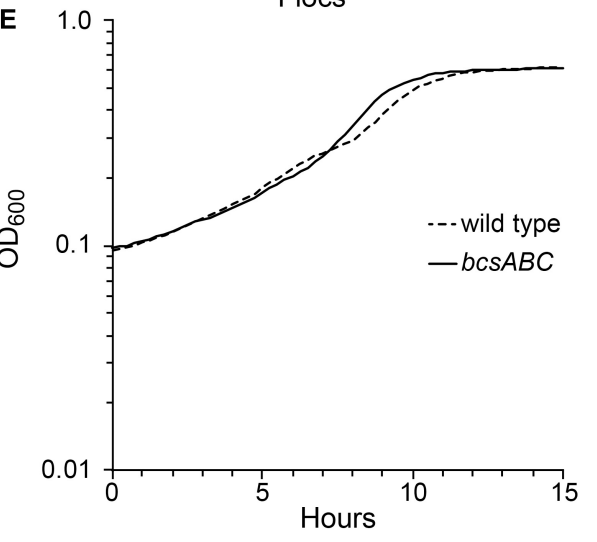

FIGURE 4 | Deletion of bcsABC operon. Non-fluorescent deletion candidates for bcs $A B C$ were isolated using the same workflow as outlined for $I d h$ and screened for deletions or wild-type (wt) alleles by PCR amplification. (A) The location of primers used to distinguish between the wt and the deletion genotype is indicated by arrows. (B,C) Agarose gel electrophoresis of products from PCR amplification of five non-fluorescent candidate colonies. (B) Amplification using primers $\mathrm{P}_{22}$ and $\mathrm{P}_{23}$ yielded a DNA fragment of $1.4 \mathrm{~kb}$ for the deletion of bCSABC (three total), while wt cells are expected to produce an amplicon of $\sim 10 \mathrm{~kb}$, which was poorly amplified under our reaction

(Continued)
FIGURE 4 | Continued

conditions. (C) To verify the genotype of wt strains, a second PCR experiment using primers P21 and P22 was included. In this case, wt strains are expected to yield an amplicon of $0.7 \mathrm{~kb}$ while the deletion strain should not produce a band. Similar results were obtained from six biological replicates of this workflow. (D) To test the phenotype of our mutant, we compared flocculation of the wt (left) and deletion strain (right) after growth for $15 \mathrm{~h}$ in glucose minimal media under aerobic conditions. Flocs are indicated by the arrow. (E) Growth curve of wt and mutant strains grown in glucose minimal media under anaerobic conditions.

one representative recombinant (PK15557) by PCR amplification across $l d h$ using a pair of primers annealing at a region further upstream and downstream of the plasmid $l d h$ targeting sequences (Figure 3D). The recombinant strain (shown in Figure 3E) also showed minor bands for both the genotype of wild type and the deletion of $l d h$ indicating that these variants were present in the population at a low level.

To identify the rare cells in the population that underwent an additional crossover to lose the integrated plasmid, we took advantage of the fact that these cells will also be non-fluorescent because of the loss of $g f p$. We grew the recombinant strain for seven cell doublings without chloramphenicol selection and then enriched for the desired population of $g f p^{-}$cells by using a fluorescence-activated cell sorter (FACS). Cells were sorted to obtain 10,000 events that were binned into the gate indicative of non-fluorescent cells (Figures 3A,B). We typically observed that viability of the sorted cells was consistently $20 \%$ or less but only those sorted into the $g f p^{-}$bin. Possibly, in the nested gate that we use for $g f p^{-}$cells, unwanted dead cells or debris of size similar to the Z. mobilis cells also appear. Nevertheless, we routinely obtain hundreds of colonies after plating the non-fluorescent cells. Visualization of the plates with an imaging system showed that $>90 \%$ of the colonies were non-fluorescent (Figure 3C).

To distinguish crossover events that regenerate wild type $l d h$ from the $l d h$ deletion, we used PCR amplification (Figures 3DF). Of 10 non-fluorescent colonies screened by PCR, 6 isolates had a DNA fragment of the size expected for the ldh deletion strain (Figures 3D-F), whereas four were wild type. We showed that the deletion endpoints were as predicted from our plasmid design by Sanger sequencing of the $l d h$ gene region (Supplementary Figure S1). Thus, because the FACS sorting step takes just a few minutes, this strategy for enriching for nonfluorescent cells and then screening non-fluorescent colonies by PCR amplification allowed rapid identification of strains containing the deletion of interest with no other markers.

\section{Deletion of $b c s A B C$ Operon}

Next, we tested whether we could efficiently delete a larger size operon, choosing the $8.3 \mathrm{~kb} b c s A B C$ operon, encoding functions for cellulose synthesis, since its function is known to be dispensable in Z. mobilis (Deutschbauer et al., 2014; Xia et al., 2018; Jones-Burrage et al., 2019). In addition, elimination of $Z$. mobilis bcs $A B C$ should minimize flocculation in liquid medium, which hinders accurate measurements of optical density $\left(\mathrm{OD}_{600}\right)$ (Figure 4). Our suicide plasmid was engineered to contain two 500 bp sequences, immediately 
flanking the $b c s A B C$ operon ( $\mathrm{pPK} 15538$ ). The plasmid was introduced by conjugation, and recombinants were confirmed by PCR. Following culturing without selection, we sorted cells to obtain 10,000 non-fluorescent events and plated for single colonies. Of five non-fluorescent colony candidates screened by PCR, three were confirmed to have the desired deletion of the $b c s A B C$ operon (Figure 4). We showed that the deletion endpoints were as predicted from our plasmid design by Sanger sequencing of the $b c s$ gene region (Supplementary Figure S1). This example shows that deletion of a longer sequence does not affect the efficiency of obtaining deletions. To determine if the strain lost the flocculating phenotype, we grew the wild type and the deletion strain in minimal medium without shaking. As expected, wild type produced flocs while the deletion strain did not produce flocs (Figure 4D). Further, growth curves with the deletion strain showed a smooth curve unlike wild type (Figure 4E).

\section{Insertion of crtIEB Genes Into the Genome}

For strain engineering, a critical tool is to be able to equip bacteria with novel pathways to produce products of interest. Thus, we also tested whether we could use our suicide plasmid to deliver heterologous genes and recombine them into the genome. An advantage of this method is that these added genes would be stably maintained on the genome and would require no added antibiotics for selection. We demonstrate this by replacing the ldh gene with the heterologous $c r t I E B$ operon (CRT) which produces an easily detected orange-pigmented carotenoid, neurosporene (Figure 5A) from R. sphaeroides (Su et al., 2018).

In this case, we placed the crtIEB genes from $R$. sphaeroides between the ldh targeting sequences on plasmid pPK15564 (Figure 5B). We also optimized RBSs (Salis et al., 2009; Borujeni et al., 2014) upstream of each gene. The plasmid was introduced by conjugation, and recombinants were confirmed by PCR. Following culturing without selection, we sorted cells to obtain 10,000 non-fluorescent events and plated for single colonies. Visualization of the plates indicated that some of the nonfluorescent colonies were also distinctly orange (Figure 5C). PCR screening of two non-fluorescent pigmented colonies confirmed the insertion of the operon at the $l d h$ gene site (Figures 5D,E). We showed that the insertion junctions were as predicted from our plasmid design by Sanger sequencing (Supplementary Figure S1). Spectral analysis of acetone-methanol extracts of these strains showed an absorbance spectrum characteristic of neurosporene (Figure 5F; Takaichi and Maoka, 2015).

\section{Optimization of Expression of Genes in Z. mobilis}

We initially encountered insufficient expression of $g f p$ in Z. mobilis for differentiating fluorescent from non-fluorescent colonies or cells. The ribosome binding site for $g f p$, originally obtained from a stable vector pRL814 was sufficient to produce fluorescent colonies from a plasmid but not when integrated
A

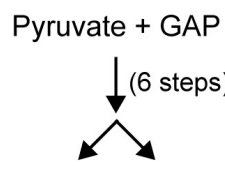

DMAPP

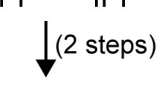

FPP

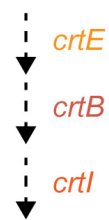

Neurosporene
B

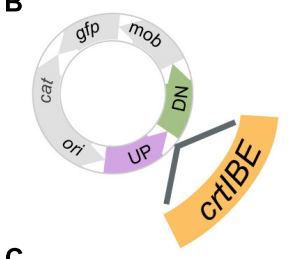

C

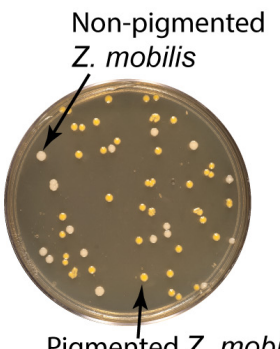

Pigmented Z. mobilis with carotenoid pathway.
D

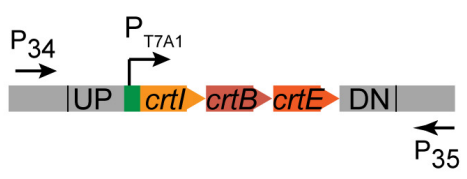

$\mathbf{E}$

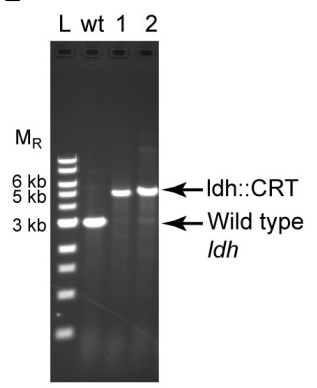

$\mathbf{F}$

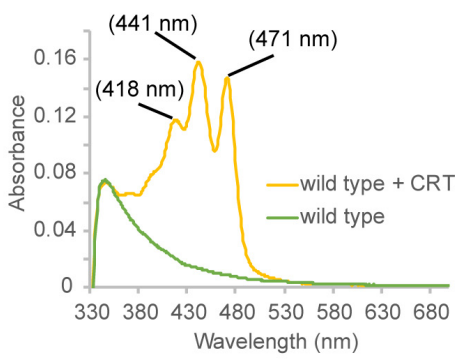

FIGURE 5 | Insertion of a functional CRT cassette. (A) A heterologous pathway for production of the carotenoid neuroporene by introduction of the crtIBE genes from Rhodobacter sphaeroides. Dotted arrows indicate the reactions mediated by the $R$. sphaeroides gene products. (B) Schematic of suicide plasmid used to deliver crtIBE to Z. mobilis Idh. UP and DN are the same 500 bp sequences used to delete the Idh gene. (C) Non-fluorescent candidates containing crtIBE replacing Z. mobilis Idh were isolated using the same workflow as outlined for ldh and screened for insertions first by the presence of orange colored colonies. (D) The location of primers used to distinguish between the wt and the insertion genotype is indicated by arrows. (E) Agarose gel electrophoresis of products from PCR amplification of orange colored colonies. Amplification using primers $\mathrm{P}_{34}$ and $\mathrm{P}_{35}$ yielded a DNA fragment of $5.4 \mathrm{~kb}$ for the insertion of crt/BE, whereas wt cells are expected to produce an amplicon of $2.9 \mathrm{~kb}$. Selected size markers are indicated on the left $\left(\mathrm{M}_{R}\right)$. (F) Absorption spectrum of acetone:methanol extracts from orange colored colonies containing crt/BE compared to wt. Peak maxima characteristic of neurosporene are marked. We examined more than five isolates for insertion of the CRT cassette in independent experiments, and measured neurosporene from three different isolates. We conducted two independent experiments both resulting in the successful insertion of the CRT cassette at the ldh locus. 

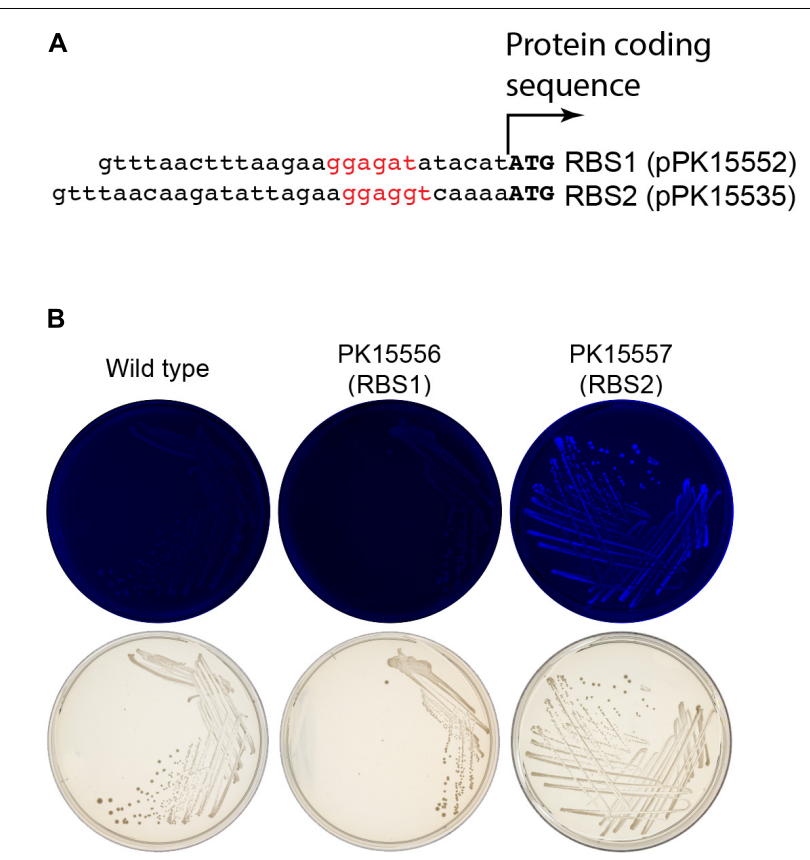

FIGURE 6 | Enhancement of gfp expression to increase colony fluorescence. (A) Comparison of the ribosome binding sites (rbs) from pPK15535 plasmid containing the optimized gfp compared to the original suicide plasmid pPK15552, in which gfp possessed a ribosome binding site from pRL814. This latter variant failed to produce detectable fluorescence in Z. mobilis in single copy. The RBS was improved using the Salis program (Salis et al., 2009; Borujeni et al., 2014). (B) Top plates show the fluorescence images of colonies from indicated integrated plasmids strains excited at $490 \mathrm{~nm}$ and imaged at $510 \mathrm{~nm}$. Bottom plates show the same colonies viewed with visible light. Only the cells possessing GFP translated from the improved RBS have detectable fluorescence.

into the genome ( $Z$. mobilis PK15556, Figure 6B). To improve the robustness of the GFP fluorescence screening, the ribosome binding site of $g f p$ was modified guided by the results from the RBS calculator for maximizing predicted RBS strength (Salis et al., 2009; Borujeni et al., 2014). The difference between the original sequences and the optimized ribosome-binding site that we used to optimize expression of $g f p$ for our suicide plasmid is shown in Figure 6A. Similar optimization of the crtIEB genes using the RBS calculator was required to detect pigmented colonies for neurosporene production (data not shown).

\section{DISCUSSION}

This work describes the development of a new flexible method for genome engineering in Z. mobilis, and how this method can be used for both knocking out genes and introducing novel sequences. We demonstrated the general utility of our genome engineering method by creating two targeted gene deletion strains and by adding a functional heterologous operon to a defined position in the $Z$. mobilis genome. The suicide plasmid and general approach should also be useful to other non-model organisms that benefit from the simple broad host range conjugation delivery system and recombinationbased strategy.

Currently, deletion of genes in $Z$. mobilis relies on recombineering strategies to replace a gene typically with an antibiotic resistance marker cassette. Removal of this marker to allow subsequent genome modification has been demonstrated by a recombination reaction using FLP recombinase (Zou et al., 2012) when the marker is flanked with FRT sequences. However, this approach is time consuming due to the additional steps needed to introduce and remove the stable plasmid encoding FLP recombinase. In addition, FRT sequences remain in the genome providing sites for unwanted recombination, which could negatively impact iterative strain design. Counter selection with $s a c B$ on sucrose media (Xia et al., 2018) is feasible but relies on the desired phenotype or the native $Z$. mobilis $s a c B$ from not negatively impacting the sucrose selection. Our approach of screening for the loss of GFP fluorescence should not negatively impact the desired phenotype as far as we are aware.

We also showed that the frequency of a single crossover event in $Z$. mobilis averages $0.3 \%$, which indicates that growth of a recombinant strain for just a few cell doublings generates sufficient cell mass to screen for a second recombination event. FACS facilitated the easy isolation of these rare events from the total population by sorting. Overall, this approach allows creation of strains containing a markerless addition or removal of a gene in two recombination events ad infinitum. This approach will be extremely useful in introducing genes for new pathways into $Z$. mobilis, which can be added at any position in the genome. By simply altering the $Z$. mobilis sequences flanking the genes of interest on the suicide plasmid, genes can be added to replace native ones, can be incorporated into native operons, or as stand alone operons, demonstrating the versatility of this approach. Our method is also not limited to FACS availability, since one can screen for non-fluorescent colonies by plating the recombinant cell suspension grown without selection and visually identify colonies that have lost the fluorescence using an imager.

\section{CONCLUSION}

In summary, our method has several highly desirable attributes; namely, it is markerless, it can be greatly accelerated with FACS, and it leverages the simple and efficient process of conjugation. Critical to this technique's success, especially for use with FACS, was our optimization of the expression of $g f p$ by RBS optimization. This enabled the visualization of fluorescent colonies and sorting of cells by FACS from a single, chromosomally inserted copy of $g f p$. We expect our method to be of value to genetic studies in the field, and our introduction of the use of FACS to enrich for desired phenotypes to be of exceptional value to $Z$. mobilis studies in the future. Finally, this method provides a simple approach for metabolic engineering where iterative strategies for genome engineering are necessary to build strains with desirable traits through sequence addition or deletion for producing molecules of interest. 


\section{DATA AVAILABILITY STATEMENT}

All datasets generated for this study are included in the manuscript/Supplementary Files.

\section{AUTHOR CONTRIBUTIONS}

PL and PK conceived the overall study. PL, PK, RL, and IG designed the experiments. PL, FW, and YL carried out the experiments. PK and PL drafted the manuscript and all authors agreed on the final version of the manuscript.

\section{FUNDING}

This work was supported by the Great Lakes Bioenergy Research Center and the United States Department of Energy, Office of Science, Office of Biological and Environmental Research under Award Numbers DE-SC0018409 and DE-FC02-07ER64494.

\section{ACKNOWLEDGMENTS}

We thank Dr. Timothy Donohue and Rachelle Lemke for providing R. sphaeroides 2.4.1 genomic DNA as a source of crtIEB

\section{REFERENCES}

Blodgett, J. A., Thomas, P. M., Li, G., Velasquez, J. E., van der Donk, W. A., Kelleher, N. L., et al. (2007). Unusual transformations in the biosynthesis of the antibiotic phosphinothricin tripeptide. Nat. Chem. Biol. 3, 480-485. doi: 10.1038/nchembio.2007.9

Bo, W., Mingxiong, H., Feng, H., Zhang, Y., Hu, Q., and Zhang, Y. (2013). Construction and characterization of restriction-modification deficient mutants in Zymomonas mobilis ZM4. Chin. J. Appl. Environ. Biol. 19, 189-197. doi: 10.3724/SP.J.1145.2013.00189

Bochner, B., Gomez, V., Ziman, M., Yang, S. H., and Brown, S. D. (2010). phenotype microarray profiling of Zymomonas mobilis ZM4. App. Biochem. Biotechnol. 161, 116-123. doi: 10.1007/s12010-009-8842-2

Borujeni, A. E., Channarasappa, A. S., and Salis, H. M. (2014). Translation rate is controlled by coupled trade-offs between site accessibility, selective RNA unfolding and sliding at upstream standby sites. Nucleic Acids Res. 42, 26462659. doi: 10.1093/nar/gkt1139

Bradley, D. E., Taylor, D. E., and Cohen, D. R. (1980). Specification of surface mating systems among conjugative drug resistance plasmids in Escherichia coli K-12. J. Bacteriol. 143, 1466-1470.

Cao, Q. H., Shao, H. H., Qiu, H., Li, T., Zhang, Y. Z., and Tan, X. M. (2017). Using the CRISPR/Cas9 system to eliminate native plasmids of Zymomonas mobilis ZM4. Biosci. Biotechnol. Biochem. 81, 453-459. doi: 10.1080/09168451. 2016.1189312

Chang, A. C., and Cohen, S. N. (1978). Construction and characterization of amplifiable multicopy DNA cloning vehicles derived from the P15A cryptic miniplasmid. J. Bacteriol. 134, 1141-1156.

Deutschbauer, A., Price, M. N., Wetmore, K. M., Tarjan, D. R., Xu, Z., Shao, W., et al. (2014). Towards an informative mutant phenotype for every bacterial gene. J. Bacteriol. 196, 3643-3655. doi: 10.1128/JB.01836- 14

Ghosh, I. N., Martien, J., Hebert, A. S., Zhang, Y., Coon, J. J., Amador-Noguez, D., et al. (2019). OptSSeq explores enzyme expression and function landscapes to maximize isobutanol production rate. Metab. Eng. 52, 324-340. doi: 10.1016/j. ymben.2018.12.008 genes; Dr. William W. Metcalf for strain WM6026; Dr. Yaoping Zhang for plasmid pSUP202; and Dr. Vatsan Raman for use of his FACS. We also thank Dr. Tomas Gverzdys, Dr. Isabel Askenasy, Dr. Erin Mettert, Dr. Yaoping Zhang, and Dr. Donna Bates for proofreading and valuable suggestions on this manuscript. We are also thankful to Laura C. Vanderploeg for helping in image capturing and making figures.

\section{SUPPLEMENTARY MATERIAL}

The Supplementary Material for this article can be found online at: https://www.frontiersin.org/articles/10.3389/fmicb. 2019.02216/full\#supplementary-material

FIGURE S1 | Sequence of the modified genome of $Z$. mobilis strains. To verify the structure of the insertion or deletion strains, we sequenced across the junction regions of genomic DNA. (A) For the Idh deletion strain, genomic DNA was amplified using primer P34 and P35 and sequenced using primer P34. (B) For deletion of bCSABC, genomic DNA was amplified using primer P22 and P23 and sequenced using primer P22. (C,D) For insertion of the CRT cassette at the $/ d h$ locus, we amplified upstream of the CRT cassette using primer P34 and P36 and sequenced using primer P34 (C) and amplified downstream of the CRT cassette using primer P37 and P35 (D) and sequenced using primer P35. Colored arrows indicate junctions of deletion or insertion of heterologous sequence. Top line represents expected sequence of the DNA fragment and the bottom line represents the sequencing data.

He, M. X., Wu, B., Qin, H., Ruan, Z. Y., Tan, F. R., Wang, J. L., et al. (2014). Zymomonas mobilis: a novel platform for future biorefineries. Biotechnol. Biofuels 7:101. doi: 10.1186/1754-6834-7-101

Jones-Burrage, S. E., Kremer, T. A., and McKinlay, J. B. (2019). Cell aggregation and aerobic respiration are important for Zymomonas mobilis ZM4 survival in an aerobic minimal medium. Appl. Environ. Microbiol. 85:10. doi: 10.1128/AEM. 00193-19

Martien, J. I., Hebert, A. S., Stevenson, D. M., Regner, M. R., Khana, D. B., Coon, J. J., et al. (2019). Systems-level analysis of oxygen exposure in Zymomonas mobilis: implications for isoprenoid production. mSystems 4:e00284-18. doi: 10.1128/mSystems.00284- 18

Pedelacq, J. D., Cabantous, S., Tran, T., Terwilliger, T. C., and Waldo, G. S. (2006). Engineering and characterization of a superfolder green fluorescent protein. Nat. Biotechnol. 24, 79-88. doi: 10.1038/nbt1172

Salis, H. M., Mirsky, E. A., and Voigt, C. A. (2009). Automated design of synthetic ribosome binding sites to control protein expression. Nat. Biotechnol. 27, 946-950. doi: 10.1038/nbt.1568

Senthilkumar, V., Rameshkumar, N., Busby, S. J., and Gunasekaran, P. (2004). Disruption of the Zymomonas mobilis extracellular sucrase gene (sacC) improves levan production. J. Appl. Microbiol. 96, 671-676. doi: 10.1111/j. 1365-2672.2003.02169.x

Simon, R., Priefer, U., and Puhler, A. (1983). A broad host range mobilization system for invivo genetic-engineering - transposon mutagenesis in gramnegative bacteria. Biotechnology 1, 784-791. doi: 10.1038/nbt1183-784

Strazdina, I., Balodite, E., Lasa, Z., Rutkis, R., Galinina, N., and Kalnenieks, U. (2018). Aerobic catabolism and respiratory lactate bypass in Ndh-negative Zymomonas mobilis. Metab. Eng. Commun. 7:e00081. doi: 10.1016/j.mec.2018. e00081

Su, A., Chi, S., Li, Y., Tan, S., Qiang, S., Chen, Z., et al. (2018). Metabolic redesign of Rhodobacter sphaeroides for lycopene production. J. Agric. Food Chem. 66, 5879-5885. doi: 10.1021/acs.jafc.8b00855

Takaichi, S., and Maoka, T. (2015). Identification and spectroscopic characterization of neurosporene. Biotechnol. Lett. 37, 2027-2031. doi: $10.1007 /$ s10529-015-1884-3 
Wang, X., He, Q., Yang, Y., Wang, J., Haning, K., Hu, Y., et al. (2018). Advances and prospects in metabolic engineering of Zymomonas mobilis. Metab. Eng. 50, 57-73. doi: 10.1016/j.ymben.2018.04.001

Wraight, C. A., Lueking, D. R., Fraley, R. T., and Kaplan, S. (1978). Synthesis of photopigments and electron transport components in synchronous phototrophic cultures of Rhodopseudomonas sphaeroides. J. Biol. Chem. 253, 465-471.

Xia, J., Liu, C. G., Zhao, X. Q., Xiao, Y., Xia, X. X., and Bai, F. W. (2018). Contribution of cellulose synthesis, formation of fibrils and their entanglement to the self-flocculation of Zymomonas mobilis. Biotechnol. Bioeng. 115, 2714-2725. doi: 10.1002/bit.26806

Yang, S. H., Fei, Q., Zhang, Y. P., Contreras, L. M., Utturkar, S. M., Brown, S. D., et al. (2016). Zymomonas mobilis as a model system for production of biofuels and biochemicals. Microb. Biotechnol. 9, 699-717. doi: 10.1111/17517915.12408
Zou, S. L., Hong, L. F., Wang, C., Jing, X., and Zhang, M. H. (2012). Construction of an unmarked Zymomonas mobilis mutant using a site-specific FLP recombinase. Food Technol. Biotechnol. 50, 406-411.

Conflict of Interest: The authors declare that the research was conducted in the absence of any commercial or financial relationships that could be construed as a potential conflict of interest.

Copyright (c) 2019 Lal, Wells, Lyu, Ghosh, Landick and Kiley. This is an open-access article distributed under the terms of the Creative Commons Attribution License (CC BY). The use, distribution or reproduction in other forums is permitted, provided the original author(s) and the copyright owner(s) are credited and that the original publication in this journal is cited, in accordance with accepted academic practice. No use, distribution or reproduction is permitted which does not comply with these terms. 